\title{
Podcast como tecnologia educacional para idosos e seus cuidadores: relato de experiência
}

\author{
Podcast as an educational technology for seniors and their caregivers: an experience report
}

Podcast como tecnología educativa para personas mayores y sus cuidadores: un informe de experiencia

\section{Resumo}

Este artigo apresenta como objetivo descrever o uso de podcast como tecnologia educacional para idosos e seus cuidadores. É uma proposta de relato de experiência, com abordagem qualitativa do tipo descritiva com vistas a divulgação de informações relevantes para idosos e seus cuidadores desenvolvido no projeto de extensão da Escola de Enfermagem Aurora de Afonso Costa pela Pró Reitoria de Extensão da Universidade Federal Fluminense. Este artigo está pautado nas seguintes discussões: a importância da rede de apoio a cuidadores de idosos; orientações sobre a pessoa idosa no período de Pandemia da Covid-19 com ênfase na vacinação e, o podcast como tecnologia educacional para idosos e seus cuidadores. Esta tecnologia possibilita uma compreensão sobre as pessoas idosas vivenciados no cotidiano no sentido de possibilitar a melhoria dos cuidados desenvolvidos visando a qualidade de vida, sobretudo durante a pandemia da Covid-19, tornando assim acessível e de baixo custo as informações imprescindíveis para a saúde do idoso. Portanto, o uso de podcast como tecnologia educacional tem uma proposta de informar e comunicar questões de destaque para idosos, cuidadores e familiares sendo uma ferramenta que permite oferecer conteúdos relacionados à saúde desse idoso e aos seus cuidadores.

Palavras-chave: Enfermagem; Pandemias; Coronavírus; Tecnologia; Educação.

\begin{abstract}
This article aims to describe the use of podcasts as an educational technology for seniors and their caregivers. It is a proposal for an experience report, with a descriptive qualitative approach, with a view to disseminating relevant information for the elderly and their caregivers, developed in the extension project of the Aurora de Afonso Costa School of Nursing by the Dean of Extension at the Federal Fluminense University. This article is based on the following discussions: the importance of the support network for caregivers of the elderly; guidance on the elderly during the Covid-19 Pandemic period, with an emphasis on vaccination and the podcast as educational technology for seniors and their caregivers. This technology enables an understanding of elderly people experienced in daily life in order to enable the improvement of care developed aiming at quality of life, especially during the Covid-19 pandemic, thus making essential information for the health of the old man. Therefore, the use of podcasts as an educational technology has a proposal to inform and communicate outstanding issues for the elderly, caregivers and family members, being a tool that allows us to offer content related to the health of this elderly person and their caregivers. Keywords: Nursing; Pandemics; Coronavirus; Technology; Education.
\end{abstract}




\begin{abstract}
Resumen
Este artículo tiene como objetivo describir el uso de podcasts como tecnología educativa para personas mayores y sus cuidadores. Se trata de una propuesta de relato de experiencia, con enfoque descriptivo cualitativo, con miras a difundir información relevante para las personas mayores y sus cuidadores, desarrollado en el proyecto de extensión de la Escuela de Enfermería Aurora de Afonso Costa por el Decano de Extensión de la Universidad Federal Fluminense. Este artículo se basa en las siguientes discusiones: la importancia de la red de apoyo a los cuidadores de ancianos; la orientación a los ancianos durante el período de la Pandemia Covid-19, con énfasis en la vacunación y el podcast como tecnología educativa para personas mayores y sus cuidadores. Esta tecnología permite conocer a las personas mayores experimentadas en la vida cotidiana con el fin de posibilitar la mejora de la atención desarrollada con miras a la calidad de vida, especialmente durante la pandemia Covid-19, haciendo accesible y baja información esencial para la salud de la población. -costo. anciano. Por tanto, el uso de los podcasts como tecnología educativa tiene una propuesta para informar y comunicar temas relevantes para las personas mayores, cuidadores y familiares, siendo una herramienta que nos permite ofrecer contenidos relacionados con la salud de esta persona mayor y sus cuidadores.
\end{abstract}

Palabras clave: Enfermería; Pandemias; Coronavirus; Tecnología; Educación.

\title{
1. Introdução
}

Com o envelhecimento da pessoa no seu processo natural da vida, é verificado a atuação mais predominante da família como suporte ao idoso, onde passam a assumir os cuidados e atenção. Em alguns momentos há também a presença de alterações comportamentais, funcionais e cognitivos pelo idoso em que o cuidador familiar tem o seu cotidiano modificado necessitando de auxílio e informações importantes para lidar com essa realidade em que muitas vezes há uma sobrecarga elevada de atividades de cuidados (INFORMASUS, 2020).

Em nosso país é uma característica importante, a família ainda representar a principal fonte de apoio esperada para o cuidado no âmbito domiciliar ao idoso, devendo receber o suporte indispensável das redes de apoio previstos nas políticas públicas de saúde como um direito (Almeida et al., 2018).

Também é notório que muitos cuidadores conseguem avançar nos cuidados ao idoso com conhecimento, destreza manual e emocional bem como um bom suporte familiar. No entanto, existem cuidadores que em sua grande maioria exercem essas atividades de forma isolada com dúvidas importantes sobre as alterações comportamentais, funcionais e cognitivos necessitando de uma rede de suporte e de esclarecimento principalmente durante a pandemia do coronavírus (INFORMASUS, 2020). Nesta realidade a atuação da rede de suporte se tornou imprescindível, principalmente com a utilização de tecnologia educacional.

A identificação do tipo de apoio ao idoso e seu cuidador (suprimento das necessidades psicobiológicas e psicossociais), relações mútuas (idoso/cuidador) voltada sentimentos de gratidão, prazer, satisfação e amor expressados e situações inerentes ao cuidador que são muitas vezes específicas (Pacheco et al, 2021) nos mostram que a atenção é redobrada para esse tipo de clientela.

Na tentativa de dar uma resolutiva a essa situação muitas famílias como fonte de suporte recorre a rede de saúde e, também das tecnologias educacionais onde procuram apoio a questões relevantes sobre: o cuidador idoso, escassa rede familiar, elevada atividade no cuidado, depressão, impacto da doença, dificuldades no desenvolvimento do cuidado e apoio emocional (Camacho et al., 2020).

Um outro aspecto relevante está no fato de considerar essa demanda de informações a este público, através de recursos digitais para divulgar conteúdos educacionais como os podcasts, que contempla a Agenda nacional de prioridades de pesquisa em saúde (ANPPS) no eixo 12 (Saúde do Idoso), subitem 12.5 (Análise da Gestão e das Práticas das Equipes no cuidado às especificidades em saúde da pessoa idosa) como fonte de comunicação e informação durante a pandemia da Covid19 (Brasil, 2018). 
Com essa constatação relevante a introdução do formato de podcast para o idosos e seus cuidadores torna-se uma tecnologia educacional positiva para informar determinados conteúdos sobre a saúde dessa população, sobre os cuidadores com vistas à melhoria de vida através do cotidiano dessa parcela da população.

Entendendo atual natureza educacional do podcast, este deve ser apoiado num caráter livre e aberto, instituído e planejado que repercute em uma evolução importante no exercício democrático, amplificador da comunicação importante para aquele que o recebe (Freire, 2017).

O podcast como recurso digital promove um alcance ao público de forma relevante, mas é importante conhecer as necessidades desta população para esta tecnologia possa de fato atender as demandas de informações específicas como por exemplo, ocorreu durante a pandemia da Covid-19, porque esta mídia tem um acesso fácil, baixo custo e com divulgação disponível a qualquer momento (Leite et al., 2020).

Desta forma, a proposta do uso de podcast como tecnologia educacional destaca-se como relevante e interativa para divulgar informações nas mídias sociais sobre o idoso e seus cuidadores com questões referentes a rede de apoio, os cuidados específicos e cotidianos bem como a importância da vacinação e outros assuntos relacionados a Covid-19. Neste sentido, a relevância e justificativa se insere no planejamento, desenvolvimento e implementação dessa tecnologia educacional para viabilizar informações importantes que visam cuidados importantes a população idosa e seus cuidadores de forma integral e colaborativa.

Desta forma, este artigo tem como objetivo descrever o uso de podcast como tecnologia educacional para idosos e seus cuidadores.

\section{Metodologia}

É uma proposta de relato de experiência, com abordagem qualitativa do tipo descritiva com vistas a divulgação de informações relevantes para idosos e seus cuidadores desenvolvido no projeto de extensão da Escola de Enfermagem Aurora de Afonso Costa pela Pró Reitoria de Extensão da Universidade Federal Fluminense.

Este relato tem uma abordagem qualitativa a partir de informações coletadas no contexto dos acontecimentos através de fenômenos sociais se caracterizando como um estudo de caso com o propósito de descrever determinadas particularidades de interesse (Pereira et al., 2018).

Neste sentido, o podcast foram desenvolvidos com conteúdos informativos sobre a Pandemia da Covid-19 e os cuidados associados a esse público principalmente aqueles com doenças crônico-degenerativas. Também foram postados conteúdos referentes a rede de apoio, a vacinação e a dose de reforço da Covid-19 previsto no programa nacional de imunização.

Estes podcasts estão hospedados no portal Educapes (https://educapes.capes.gov.br/) e também divulgados no site educativo sobre os cuidados de enfermagem desenvolvidos a pessoa com Doença de Alzheimer e outros transtornos demenciais (http://cuidadosalzheimer.uff.br) que faz parte do projeto de extensão através do blog educativo (http://cicacamacho.blogspot.com/) que divulga informações relevantes acerca dos cuidados de enfermagem a esta clientela. Com a presença da Pandemia da Covid-19 instalada desde março de 2020, de 78.000 visualizações no blog foi apresentada até a presente data 88.521 visualizações, ou seja, mais de 10.000 acessos do público-alvo.

Este artigo está pautado nas seguintes discussões: a importância da rede de apoio a cuidadores de idosos; orientações sobre a pessoa idosa no período de Pandemia da Covid-19 com ênfase na vacinação e, o podcast como tecnologia educacional para idosos e seus cuidadores. 


\section{Resultados e Discussão}

\section{A importância da rede de apoio a cuidadores de idosos}

Um dos aspectos de relevância nas informações elencadas aos idosos e seus cuidadores são relacionadas ao processo de envelhecimento e a necessidade do autocuidado. Esses aspectos são fonte de análise para os profissionais de saúde que determinam quais os principais déficits de autocuidado e que tendem a se elevar na presença de outras comorbidades.

Com o avanço da idade do idoso há uma situação de crise inesperada no seio familiar, gerando modificações na rotina dos membros, especialmente na vida do familiar responsável pelo papel de prestar o cuidado. Para melhor assistir essa família é necessário avaliar o efeito da modificação de papéis que passaram por transições advindas da experiência de cuidar. Além disso, é preciso considerar que com essas modificações a rotina pessoal compromete o processo de transição do familiar, pois a obrigação de conciliar os compromissos diários com as atividades relacionadas ao acompanhamento do idoso tende a gerar muita angústia na conciliação dessas atividades cotidianas (Nunes et al., 2019)

Portanto, para melhor compreensão faz-se necessário entender sobre as redes de apoio social, assim como o suporte social, para o atendimento as necessidades de cuidado do idoso. A rede de apoio social é um conjunto de relacionamentos de um indivíduo ou de elos entre um conjunto de pessoas, já o suporte social se relaciona a qualidade das interações e como estas ocorrem (Ribeiro \& Paulim, 2014).

É mister que as necessidades cotidianas dos cuidadores familiares se referem a insegurança relacionada a ausência de orientação nos aspectos geriátricos e gerontológicos; repercussões psicossociais pelo trabalho penoso e repetitivo; privação de contato social em virtude das elevadas demandas de cuidado; dificuldades na conciliação do cuidado e o trabalho; suspensão da vida profissional e dificuldades financeiras importante. Há um aumento da vulnerabilidade dos cuidadores idosos frente à carga de trabalho rotineira e permanente (Sousa et al., 2021).

Durante a pandemia da Covid-19 surgiu uma preocupação relevante em relação aos diversos arranjos familiares em que a pessoa idosa está inserida como a independência e autonomia do idoso nas suas atividades cotidianas, a carga de estresse, com à insegurança e incerteza de um contexto de pandemia em que o maior risco de vulnerabilidade foi atribuída aos idosos (Petermam et al., 2020).

Além disso, em nosso país existem em quantitativo considerável idosos que vivem em condições difíceis e ainda promovem o sustento da família em virtude do elevado desemprego, estão acamados e com mobilidade reduzida, com síndromes demenciais, vivem sozinhos, dentre outras tantas realidades (Petermam et al., 2020).

A estrutura de atendimento aos idosos no âmbito social e de saúde são precárias e, para a maioria deles, a família é a única fonte de provisão de apoio e ajuda. A resolutiva estaria centrada numa rede de suporte diversificada para tentar atender a insuficiência do suporte social do poder público (Sant' Ana \& D’Elboux, 2019a).

Deve-se levar em consideração o crescimento da população idosa e os problemas de saúde do cuidador familiar causados pela prática do cuidado ao idoso, onde os profissionais da saúde devem identificar as necessidades do cuidador como também o potencial das redes sociais possibilitando, assim, a criação de vínculos de confiança que pode contribuir para a assistência adequada dessas pessoas através de informações relevantes que causam impacto no seu cotidiano. A rede de suporte social é fundamental para que o cuidador tenha acesso a este no processo de adoecimento, planejando o cuidado do idoso no domicílio, como centro, porém o cuidador também deve ser assistido considerando a responsabilidade no processo desse cuidado (Kobayasi et al., 2019).

É fundamental a compreensão do conteúdo sobre o estilo de vida na velhice na rede de suporte, para o desenvolvimento de iniciativas de prevenção e intervenção, para à promoção e à reabilitação nos vários contextos da vida, além de proporcionar a avaliação e o planejamento de serviços e políticas destinados a promover o bem-estar de idosos (Camacho et al., 2021). 
Uma experiência de apoio virtual também é significativa porque demonstra que as tecnologias são recursos importantes e utilizados para o acesso à informação e às orientações e sobre o cuidado ao familiar, acentuadas com o distanciamento físico durante a Pandemia do coronavírus. As vivências comuns relatadas fortaleceram os vínculos entre eles e podem ser legitimadas como um espaço de escuta e acolhimento seguro e empático diminuindo o sentimento de isolamento social durante a pandemia da COVID-19 (Mattos et al., 2021).

Neste tipo de atividade educacional a participação das famílias que realizam cuidados aos idosos fortalece as redes de apoio dos mais velhos e os cuidadores e encoraja trocas sociais satisfatórias de suporte, auxiliando na melhoria do desempenho cognitivo e funcional do idoso, o que pode ter um efeito positivo impacto na saúde dos cuidadores e na qualidade dos cuidados oferecidos (Pavarini et al, 2021).

Outrossim, a utilização de tecnologia cria estratégias e novas possibilidades de abordagem na rede de apoio com ações mais objetivas e que promovem a comunicação necessária de acordo com as necessidades de orientação causando um impacto importante no emocional e bem-estar de cuidadores (Mattos et al., 2021).

Nessa realidade existe a importância de estudos que forneçam informações de atenção e apoio a famílias e seus cuidadores de acordo com as especificidades regionais para poder ter uma análise mais acurada sobre a associação entre suporte percebido e recebido e a expectativa de cuidado verificando as variáveis sociodemográficas, condições de saúde e funcionalidade dos idosos (Sant' Ana \& D'Elboux, 2019b).

\section{Orientações sobre a pessoa idosa no período de Pandemia da Covid-19 com ênfase na vacinação.}

As orientações para a pessoa idosa no período de pandemia sobre a vacinação contra a COVID-19 é uma das formas importantes de reduzir a mortalidade e de dar visibilidade como estratégia importante de promoção e proteção da saúde dos idosos (Souto \& Kabad, 2020).

A vacinação é um excelente meio de prevenção de doenças com normatizações relevantes previstos no programa nacional de imunização brasileiro, especialmente para o enfrentamento da pandemia do coronavírus, com vistas a benefícios para o indivíduo e para a comunidade. Portanto, a vacinação é uma estratégia de controle de doenças pensada e implementada para ter alcance populacional, podendo reduzir a reprodução do vírus, desencadeando um adoecimento mais leve e potencialmente reduzindo a capacidade do indivíduo de transmitir a doença (Souto \& Kabad, 2020).

A Sociedade Brasileira de Geriatria e Gerontologia (SBGG) destaca que a vacinação de idosos pode reduzir a mortalidade, principalmente com comorbidades e idosos com 80 anos ou mais que possuem vulnerabilidade física, sendo então essencial a realização das ações de distanciamento e isolamento social (SBGG, 2021).

Pensando nestes esclarecimentos o desenvolvimento de podcasts como tecnologia educacional traz informações confiáveis e com esclarecimento sobre a importância e segurança da vacina, auxiliando tanto no enfrentamento da pandemia de COVID-19 como na educação em saúde para idosos, seus cuidadores e familiares.

Neste sentido, foi adotada a administração, a partir do dia 15 de setembro de 2021, uma dose de reforço da vacina para todos os idosos em conformidade com o plano nacional de operacionalização da vacinação contra a Covid-19. Foi considerada a elevada cobertura vacinal com 2 doses na população idosa no Brasil, e com indícios de ascensão nas taxas de hospitalizações desta população e a possibilidade de se elevar as formas graves da Covid-19, o Mistério da Saúde iniciou a campanha da terceira dose de reforço da vacina contra a Covid-19 (Brasil, 2021).

Considerando os aspectos relevantes e a divulgação de informações equivocadas sobre a vacinação entende-se que as tecnologias educacionais voltadas aos cuidadores/familiares de idosos trouxeram uma procura de informações relacionadas aos cuidados efetuados na população idosa durante a Pandemia da Covid-19 por meio das mídias sociais despertando interesse por 
conhecimentos sobre essa enfermidade bem como a compreensão dos direitos vinculados as políticas públicas de saúde, e a relevância da vacinação contra a Covid-19.

\section{O podcast como tecnologia educacional para idosos e seus cuidadores}

Podemos definir que o podcasts é um arquivo digital de áudio, que pode ser disponibilizado via on-line, sendo este uma música e falas de determinados conteúdos e ações em inúmeras situações e momentos do dia a dia. É uma miniaturização dos dispositivos de áudio, bem como a incorporação de funções de tocador de MP3 (Freire, 2017).

No caso dos podcasts, sem dúvida com a facilidade tecnológica de gravação e produção podem ser entendidas como potencializadora de uma diversidade de opções na produção de conteúdo, em todas as etapas envolvidas. Essas mudanças nos últimos anos nos permitiram alcançar a divulgação de conteúdo científico com uma fluência que talvez fosse impensável antes (Parreiras \& Lacerda, 2021)

O desenvolvimento de podcasts permite que novas estratégias de informação e comunicação sejam oferecidas de maneira a atenuar questões relacionadas a pessoa idosa de forma educativa e de fácil acesso, com o intuito de construir conhecimentos, promover o diálogo, relacionados aos temas disponibilizados. (Leite et al., 2020).

A comunicação e a deteç̧ão precoce das necessidades mostram a necessidade da identificação dos fatores facilitadores e dificultadores que estão presentes na vida do familiar cuidador e que podem levá-lo em direção ao bem-estar, a caminho da transição saudável, ou ao risco de vivenciar uma transição difícil (Nunes et al., 2019).

Com essa necessidade nos tempos atuais vemos a amplitude e a maleabilidade espacial e temporal do podcast subsidiada por necessidades educativas específicas em que uma das principais potencialidades é a intenção de manter um exercício democrático, de baixo custo e que promove o encontro das falas e ideias (Freire, 2017).

Muitas vezes as famílias na situação de cuidado ao idoso não possuem outra opção para dividir essas atividades com outros membros da família e adicional a essa realidade há a falta de condições financeiras para contratar cuidador formal. Desta forma, conhecer desse público e compreender a motivação para o cuidar da pessoa idosa é importante não só para identificar suas necessidades e anseios, mas também para buscar meios de promover sua saúde e bem-estar (Almeida et al., 2018).

Nesse contexto, a elaboração de tecnologias educacionais corrobora para um meio interativo e cooperativo de acordo com as necessidades acerca da população idosa durante a pandemia. As tecnologias educacionais em saúde são recursos importantes, possibilitando a construção do conhecimento potencializando as informações adquiridas pelos visitantes dessa tecnologia educacional, sendo um instrumento de educação em saúde (Camacho et al., 2020).

Os profissionais de saúde devem orientar os cuidadores com as demandas de cuidados e suas especificidades decorrentes da condição dos idosos para diminuir a sobrecarga, o peso e o estresse favorecendo o aumento da qualidade de vida dos cuidadores e de seus familiares doentes (Nunes et al., 2018). Pensando nesse aspecto e com as demandas da Pandemia do Covid-19 a criação dos podcasts como tecnologia vislumbra no atendimento as necessidades de informação de forma a atenuar a sobrecarga cotidiana.

Os Podcasts como tecnologia permite o compartilhamento informações de saúde para idosos na pandemia da COVID19, mostrando-se uma maneira de levar informações de saúde aos ouvintes, de forma acessível, abrangente e com um baixo custo, beneficiando não só aos idosos, mas a toda população adulta, tornando-se assim um instrumento relevante para a educação em saúde (Silva et al, 2021).

As tecnologias educacionais incentivam o acesso as informações para os cuidadores/ familiares com acesso simples e prático, além de permitir ações vinculadas a promoção da educação em saúde, junto a essa população durante a Covid-19 (Camacho et al., 2020). 
É importante estimular essas estratégias e na maneira de abordagem dos usuários, a partir do uso de novas tecnologias educacionais nas ações educativas na área da saúde a fim de incentivar mudança do estilo de vida e melhoria das condições de saúde (Santos et al., 2020), principalmente de idosos e seus cuidadores.

Essas mídias como tecnologia educacional na área da saúde permite a compreensão dos direitos vinculados as políticas públicas de saúde, cuidados cotidianos, e questões sobre a Covid-19 vinculadas aos idosos e seus familiares, sobretudo, questões relacionadas ao isolamento social, importância da higienização das mãos, informações sobre atividades lúdicas para a pessoa idosa, assim como a relevância da vacinação contra a Covid-19 e da influenza (Camacho et al., 2021).

É preciso focar nos membros familiares que cuidam da pessoa idosa dependente para direcionar o planejamento do suporte dos serviços de saúde e garantir que os cuidadores familiares não se sintam abandonados, devendo ser compartilhado entre a família, o Estado e a sociedade civil (Sousa et al., 2021).

\section{Conclusão}

Este artigo apresenta como objetivo descrever o uso de podcast como tecnologia educacional para idosos e seus cuidadores. É destaque as múltiplas possibilidades de informação e comunicação ao idoso e seus familiares através das múltiplas redes de apoio que condicionam a sua atuação em detrimento as especificidades e necessidades de cada família que realiza o cuidado ao idoso no cotidiano.

Com o advento da pandemia da Covid-19 e o isolamento social os podcasts se tornaram ferramentas valiosas junto com outras mídias sociais no auxílio as informações de cuidados específicos a esse público, principalmente no que diz respeito as comorbidades e a campanha de vacinação contra a Covid-19. Portanto, a veiculação de informação atenuam angústias e fomentam novas oportunidades de discussão para as redes de apoio.

Como limitação do estudo, destacamos a dificuldade de acesso da população idosa e seus familiares ao acesso as tecnológicas e à internet, que limitam o alcance da maior parte desse público. É necessário, portanto, uma inclusão digital dessa população, principalmente, no que tange o acesso às redes sociais, bem como as políticas públicas de saúde e o acesso aos seus direitos de assistência pelo Estado. Diante disso, sugere-se o desenvolvimento de estudos experimentais com a finalidade de avaliar o impacto do uso contínuo do podcast na qualidade de vida dos idosos e seus cuidadores.

Essa atividade favorece o desenvolvimento de novos projetos com novas tecnologias aliado a questões pertinentes à pessoa idosa e ao seu cotidiano, seguindo as novas tendências digitais e possibilitando um alcance expressivo para os assuntos que envolvem a pessoa idosa. Existe também a importância de aplicar de fato as políticas públicas que incluam as necessidades e o suporte aos cuidadores, em especial, os familiares de pessoas idosas, a fim de melhorar o estilo vida dos cuidadores familiares, bem como a qualidade dos cuidados prestados às pessoas idosas.

\section{Referências}

Almeida, L. P. B., Menezes, T. M. O., Freitas, A. V. S., \& Pedreira, L. C. (2018). Características sociais e demográficas de idosos cuidadores e motivos para cuidar da pessoa idosa em domicílio. Rev Min Enferm, 22:e-1074

Brasil. Ministério da Saúde. (2021). Plano nacional de operacionalização da vacinação contra a Covid-19. Ministério da Saúde. https://www.gov.br/saude/pt$\mathrm{br} /$ coronavirus/publicacoes-tecnicas/guias-e-planos/plano-nacional-de-vacinacao-covid-19

Brasil. Ministério da Saúde. (2018). Agenda nacional de prioridades de pesquisa em saúde (ANPPS). Ministério da Saúde. http://bvsms.saude.gov.br/bvs/publicacoes/agenda_prioridades_pesquisa_ms.pdf

Camacho, A. C. L. F., Silva, J. O. N., Ferraz, V. H. G., \& Souza, V. M. F. (2021). Site educativo como tecnologia da informação e comunicação sobre o idoso com demência. Research, Society and Development, 10(8), e58410817651.

Camacho, A. C. L. F., Silva, R. T., \& Souza, V. M. F. (2020). Tecnologia da informação ao idoso em tempos de COVID-19. Research, Society and Development, 9(6), e124963497. 
Freire, E. P. A. (2017). Podcast: breve história de uma nova tecnologia educacional. Educação em Revista, 18(2): 55-70.

INFORMASUS. Universidade Federal de São Carlos (2020). Grupo de apoio virtual a cuidadores familiares de idosos com demências é oferecido pela ABRAz. INFORMASUS (UFSCAR), https://www.informasus.ufscar.br/grupo-de-apoio-virtual-a-cuidadores-familiares-de-idosos-com-demencias-eoferecido-pela-abraz/

Kobayasi, D. Y., Rodrigues, P. R., Fhon, S. J., Silva, L. M., Souza, A. C., \& Chayamiti, C. E. (2019). Sobrecarga, rede de apoio social e estresse emocional do cuidador do idoso. Av Enferm, 37(2):140-148.

Leite, Y. S. C. O., Rosa, K. R., Souza, M. C. B., Lima, D. B., \& Brito, T. R. P. (2020). A utilização de podcasts para a conscientização sobre violência contra a pessoa idosa: relato de experiência. Revista Extensão \& Cidadania, 8(14): 303-15.

Mattos, E. B. T., Francisco, I. C., Pereira, G. C., \& Novelli, M. M. P. C. (2021). Grupo virtual de apoio aos cuidadores familiares de idosos com demência no contexto da COVID-19. Cadernos Brasileiros de Terapia Ocupacional, 29:e2882.

Nunes, S. F. L., Alvarez, A. M., Costa, M. F. B. N. A., \& Valcarenghi, R. V. (2019). Fatores determinantes na transição situacional de familiares cuidadores de idosos com Doença de Parkinson. Texto Contexto Enferm, 28:e20170438.

Nunes, D. P., Brito, T. R. P., Duarte, Y. A. O. \& Lebrão, M. L. (2018). Cuidadores de idosos e tensão excessiva associada ao cuidado: evidencias do estudo SABE. Rev. Bras Epidemiol, 21(suppl.2): E180020.

Pacheco, E. S., Rocha, K. A., Mota, M. S., Silva, V. R., Gomes, A. T., Viana et al. (2020). Perceptions of elderly caregivers about the act of caring. Research, Society and Development, 9(7): 1-16, e283974161.

Parreiras, C. \& Lacerda, P. (2021). Tecnologia, educação e divulgação científica em antropologia: usos, consumos e produção de podcasts. Novos Debates, 7(1): E7114.

Pavarini, S. C. I., Ottaviani, A. C., Bregola, A. G., Fraga, F. J., Chagas, M. H. N., Oliveira, N. A. et al (2021). Association between perceived social support and better cognitive performance among caregivers and non-caregivers. Rev Bras Enferm,74(Suppl 2):e20200329.

Pereira, A. S, Shitsuka, D. M., Parreira, F. J., \& Shitsuka, R. (2018). Metodologia da pesquisa científica. UFSM, NTE.

Petermann, X. B., Miolo, S. B., \& Kocourek, S. (2020). Pandemia de Covid-19, Saúde do Idoso e Rede de Apoio Familiar: uma interface necessária. Revista Kairós-Gerontologia, 23(Esp.28): 449-460.

Ribeiro, F. L., \& Paulim, G. S. T. (2014). Papéis Ocupacionais e Rede de Apoio Social de Idosas em Institucionalização Permanente e Residentes na Comunidade. Revista Família, Ciclos de Vida e Saúde no Contexto Social, 2(3): 123-131.

Sant' Ana, L. A. J., \& D'Elboux, M. J. (2019a). Comparação da rede de suporte social e a expectativa para o cuidado entre idosos em diferentes arranjos domiciliares. Rev. Bras. Geriatr. Gerontol. 2019;22(3):e190012.

Sant’ Ana, L. A. J., \& D’Elboux, M. J. (2019b). Suporte social e expectativa de cuidado de idosos: associação com variáveis socioeconômicas, saúde e funcionalidade. Saúde Debate, 43(121):503-519.

Santos, L. H. R., et al. (2020). Grupo educativo e qualidade de vida de idosos hipertensos e diabéticos. Research, Society and Development, 9(1), e178911893.

SBGG. Sociedade Brasileira de Geriatria e Gerontologia. (2021). Congresso Brasileiro de Geriatria debate a importância das vacinas. SBGG, https://sbgg.org.br/congresso-brasileiro-de-geriatria-debate-a-importancia-das-vacinas/

Silva, A. S. et al (2021). Podcasts: compartilhando informações de saúde para idosos em tempos de COVID-19. Brazilian Journal of Health Review, 4(2):6312-6320.

Souto, E. P. \& Kabad, J. (2020). Hesitação vacinal e os desafios para enfrentamento da pandemia de COVID-19 em idosos no Brasil. Rev. Bras. Geriatr. Gerontol., 23(5):e210032.

Sousa, G. S. S., Silva, R. M., Reinaldo, A. M. S., Soares, S. M., Gutierrez, D. M. D., \& Figueiredo, M. L. F. (2021). "A gente não é de ferro”: Vivências de cuidadores familiares sobre o cuidado com idosos dependentes no Brasil. Ciência \& Saúde Coletiva, 26(1):27-36. 